\title{
Relationship of umbilical coiling index and cord twist direction with adverse perinatal outcomes
}

\author{
Pratibha S. Sarkate ${ }^{1 *}$, Sujitkumar Hiwale ${ }^{2}$
}

\begin{abstract}
${ }^{1}$ Department of Obstetrics and Gynecology, Malla Reddy Institute of Medical Sciences, Hyderabad, Telangana, India ${ }^{2}$ Philips Research, Bangalore, Karnataka, India
\end{abstract}

Received: 15 April 2019

Revised: 12 August 2019

Accepted: 16 August 2019

*Correspondence:

Dr. Pratibha S. Sarkate,

E-mail: dr_pratibha@outlook.com

Copyright: () the author(s), publisher and licensee Medip Academy. This is an open-access article distributed under the terms of the Creative Commons Attribution Non-Commercial License, which permits unrestricted non-commercial use, distribution, and reproduction in any medium, provided the original work is properly cited.

\begin{abstract}
Background: The main objectives of this study were to examine - (1) relationship of pregnancy-related factors (maternal age, gestational diabetes mellitus, pregnancy-induced hypertension, oligohydramnios, small for gestational age (GA), and fetal gender) and postnatally measured umbilical coiling index (UCI); (2) association of UCI and cord twist directions with the following adverse perinatal outcomes, meconium staining of amniotic fluid, non-reassuring FHR on CTG, low Apgar score (<7) at one and five minutes, low birth weight, and NICU admission.

Methods: The inclusion criteria were singleton live-birth pregnancy with GA > 34 weeks. The cases were categorized in hypocoiled (UCI $<10^{\text {th }}$ percentile), hypercoiled (UCI $>90^{\text {th }}$ percentile) and normocoiled groups. To study relationship of pregnancy-related factors and UCI multivariate logistic regression was used; whereas bivariate analysis was used to study impact of UCI on various adverse perinatal outcomes. UCI was measured by a single observer.

Results: In total, 100 subjects were enrolled. The mean UCI was $0.268\left(\mathrm{SD}=0.13 ; 10^{\text {th }}\right.$ percentile $=0.139 ; 90^{\text {th }}$ percentile $=0.410$ ) coils $/ \mathrm{cm}$. Pregnancy-related factors had non-significant relationship with UCI. For adverse perinatal outcomes, only the non-reassuring/abnormal FHR patterns were significantly associated with hypercoiled groups $(\mathrm{OR}=4.5 ; \mathrm{CI}=1.15-17.58)$. Both the cord directions had almost equal distribution without any significant difference in outcomes; however, anticlockwise twisted cords were found to have significantly high UCI.

Conclusions: No significant relationship was observed with pregnancy-related factors and UCI. However, it was observed that hypercoiled cords had significant association with non-reassuring/abnormal FHR patterns on CTG.
\end{abstract}

Keywords: India, Perinatal outcomes, UCI, Umbilical coiling index, Umbilical cord

\section{INTRODUCTION}

As intrauterine survival and wellbeing of a fetus depends on a proper exchange of nutrients and metabolites through an umbilical cord, lesions or pathologies of the umbilical cord are known to have detrimental effects on the fetus. Therefore, researchers have tried to investigate possible relationship between umbilical cord characteristics such as cord length, thickness, coiling and twist direction with various perinatal outcomes. Out of these characteristics, umbilical cord coiling, expressed in umbilical cord index (UCI), is one of the most studied parameters. A number of studies have shown a relationship with abnormal cord coiling detected either prenatally or postnatally, and cord twist directions with a number of adverse perinatal outcomes. ${ }^{1-5}$ However, there is still no consensus on an exact role of cord characteristics in adverse perinatal outcomes, as a few 
studies have also reported statistically non-significant association between cord coiling patterns and perinatal outcomes. $^{6}$

Even though a number of pregnancy-related factors are hypothesized to be associated with origin of abnormal cord coiling, it is still not fully understood whether abnormal coiling is actually a cause of pathology, or merely one of the sequelae, or both. ${ }^{1,7-9}$ Conflicting results from different studies have made it even more complicated. Furthermore, it has been noted that many studies on this topic have had several inconsistencies, such as non-consistent definitions of abnormal coiling and defining reference ranges for coiling without considering potential errors or the possible effect of gestational age into account. ${ }^{7,10}$ It is further indicated that not performing multivariate statistical analysis is also a limiting factor in many studies. ${ }^{1}$ These inconsistencies make it difficult to infer properly from the studies published in the past.

Unfortunately, with a few exceptions, not much work has been published from India on this particular topic. ${ }^{8,11-14}$ Variations in study design, and non-consistent definitions makes it difficult to draw a valid inference from these studies. Moreover, none of these studies have used multivariate logistic regression analysis, further limiting their statistical validity. Considering this scenario and given a fact that controversy still exists on both origin and significance of abnormal cord coiling, this study had two main objectives- (1) to examine relationship of pregnancy-related factors, which are hypothesized with origin of abnormal cord coiling; and (2) to study association of abnormal cord coiling and cord twist direction with adverse perinatal outcomes in a systematic manner.

\section{METHODS}

This observational prospective study was conducted in a department of obstetrics and gynaecology of a teaching hospital in India, from November 2015 to May 2016. The inclusion criteria were a live-birth singleton pregnancy with gestational age $>34$ weeks. Cases with a suspected fetal malformation or anomaly were excluded. The study was conducted in accordance with local regulations after receiving written approval of an ethical committee. Signed informed consent was obtained from all the subjects. In total, 100 subjects were enrolled for this study.

\section{Measurement of umbilical cord characteristics}

During study, postnatal UCI and umbilical cord twist directions were measured immediately after delivery of a fetus. Milking of the umbilical cord was avoided as it is known to impact the UCI. An umbilical cord was measured through its entire length from the placental to the fetal end; length of the stump of the baby was added to get total length of the cord. A complete 360-degree spiral loop of umbilical vessels around Wharton's jelly was considered as one coil; complete spirals were calculated from the neonatal end towards the placental end of the cord. UCI was defined as the total number of completed spirals in an umbilical cord divided by its length in centimetres. ${ }^{15}$ Clockwise (right/dextral) or anticlockwise (left/sinistral) direction of cord coiling was decided in relation to the neonatal end.

To avoid inter-observer variations in measurement of UCI and coiling direction, all the subjects were examined by a single observer.

\section{Categorization of cases based on UCI}

For defining the UCI categorizes, the criteria defined by Rana et al, was used. ${ }^{16}$ All the cases were ranked in an ascending order based on their UCI. The subjects with UCI below the tenth percentile were categorized as having hypocoiled cord. The subjects with UCI between the $10^{\text {th }}$ and $90^{\text {th }}$ percentiles were categorized as having normocoiled cord; whereas the subjects with UCI above the $90^{\text {th }}$ percentile were categorized as having hypercoiled cord.

\section{Pregnancy-related factors associated with abnormal cord coiling}

The following pregnancy-related factors, maternal age, gestational diabetes mellitus (GDM), pregnancy-induced hypertension (PIH), oligohydramnios (amniotic fluid index $<8 \mathrm{~cm}$ ), small for gestational age (SGA) fetuses, and fetal gender were studied to assess their impact on UCI. The SGA (birth weight $<10^{\text {th }}$ percentile for gestational age) cases were identified using customized percentile charts by Mikolajczyk et al, for Indian population. ${ }^{17}$ Multivariate logistic regression analysis was used to compute adjusted odds ratio (OR) to study association of these factors with hypocoiled and hypercoiled cord groups with normocoiled cord group as a reference.

\section{Cord characteristics and adverse perinatal outcomes}

Abnormal coiling is shown to be associated with an array of adverse perinatal outcomes. Following outcomes were studied to find out impact of UCI and cord twist directions on them, meconium staining of the amniotic fluid, low Apgar score (<7) at one and five minutes, low birth weight (LBW) neonates (birth weight $<2500 \mathrm{~g}$ ), nonreassuring fetal heart rate (FHR) patterns on cardiotocography (CTG) and admission of a neonate to a neonatal intensive care unit (NICU). The CTG was classified in normal and non-reassuring category using the National Institute for Health and Care Excellence (NICE) guidelines on intrapartum care. ${ }^{18}$ A relationship between the three UCI categories and the perinatal outcomes was examined by computing OR along with $95 \%$ confidence interval $(\mathrm{CI})$. 


\section{Statistical analysis}

To study relationship of UCI with maternal age and gestational age, the Spearman's rank correlation coefficient (rho) was used. Student's independent sample t-test and One-way Analysis of Variance (ANOVA) test were used to compare continuous variables across the different categories. The chi-squared and Fisher's exact test were used for analysis of categorical data. For all comparisons, a p-value $<0.05$ was considered as a statistically significant difference.

To study association of pregnancy-related factors and the UCI categories, multivariate logistic regression analysis was used; whereas bivariate analysis was used to study impact of the UCI categorizes on various adverse perinatal outcomes. All statistical analyses were performed using Microsoft Office Excel-2013 and Epi Info (version -7) software.

\section{RESULTS}

In total, 100 subjects were included in this study. The mean age of mothers in our study was 23.44 years with a range from 18 to 34 years. The nulliparous women constituted $52 \%$ of the study population. The median gestational age of the study population was 38.2 weeks (range, 35.3 to 40.4 weeks), with 16 preterm cases. The mean birth weight of the study population was $2761.14 \mathrm{~g}$ with a range of $1900 \mathrm{~g}$ to $3860 \mathrm{~g}$; LBW neonates constituted $26 \%$ of the study population, whereas three neonates had birth weight more than $3500 \mathrm{~g}$. Seven cases were suffering from GDM; 14 cases had PIH, whereas two cases had both GDM and PIH. The important demographic characteristics of the study population as per the UCI categories are summarized in Table 1. All the three UCI category-based groups were found to be comparable with each other in regard to maternal age, gestational age, birth weight and other parameters without any significant difference.

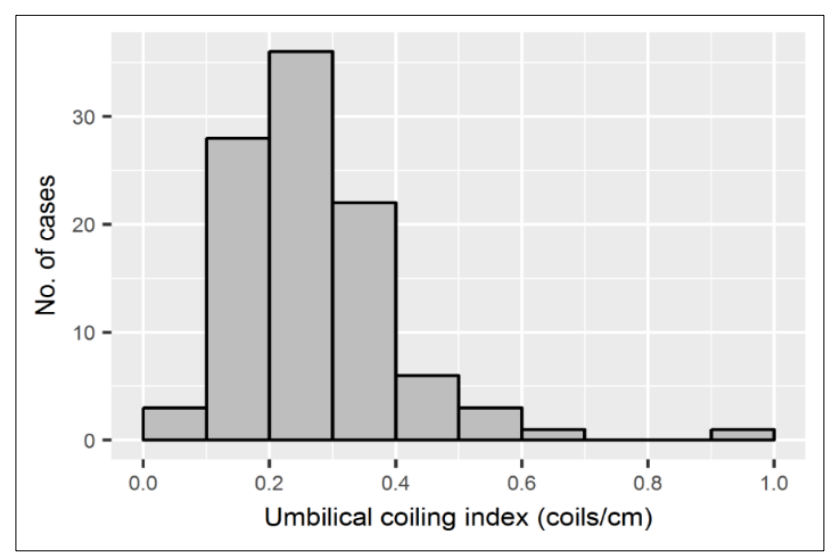

Figure 1: Histogram of umbilical coiling indices.

\section{Umbilical cord characteristics}

We observed a wide variation in length of umbilical cords with mean length of $53.25 \pm 12.22 \mathrm{~cm}$ (range, 31 to $86 \mathrm{~cm}$ ). Number of coils in umbilical cords also showed a wide variation with a range of 1 to 44 coils; the mean number of coils was $13.94 \pm 6.92$ with median and mode of 13. It was observed that length of umbilical cords and number of coils were very weakly correlated (Pearson's correlation coefficient $=0.291$ ). The mean UCI in our study was $0.268 \pm 0.13$ coils $/ \mathrm{cm}$ with median of 0.242 coils $/ \mathrm{cm}$, and a range between 0.019 to $0.911 \mathrm{coils} / \mathrm{cm}$. The values for the 10th and the 90th percentile were 0.139 and 0.410 coils $/ \mathrm{cm}$ respectively with an interquartile range equal to 0.138 . Figure 1 , shows frequency distribution of UCI.

Table 1: Demographic characteristics of the study population as per the UCI categories.

\begin{tabular}{|llllll|}
\hline Characteristic & $\begin{array}{l}\text { Study } \\
\text { population }\end{array}$ & $\begin{array}{l}\text { Hypocoiled } \\
\text { group }(\mathrm{n}=10)\end{array}$ & $\begin{array}{l}\text { Normocoiled } \\
\text { group }(\mathbf{n}=80)\end{array}$ & $\begin{array}{l}\text { Hypercoiled } \\
\text { group }(\mathbf{n}=10)\end{array}$ & $\begin{array}{l}\text { Statistical } \\
\text { difference }\end{array}$ \\
\hline Maternal age (year) & $23.44(3.11)$ & $23.8(2.20)$ & $23.36(3.23)$ & $23.7(3.17)$ & NS \\
\hline Gestational age (week) & $38.21(1.20)$ & $37.95(1.75)$ & $38.21(1.16)$ & $38.45(0.97)$ & NS \\
\hline Birth weight (g) & $2761.14(401.65)$ & $2893(387.36)$ & $2753.32(398.8)$ & $2691.1(450.57)$ & NS \\
\hline GDM & 7 & 0 & 6 & 1 & NS \\
\hline PIH & 14 & 1 & 11 & 2 & NS \\
\hline Oligohydramnios & 21 & 0 & 20 & 1 & NS \\
\hline SGA neonates & 6 & 1 & 4 & 1 & \\
\hline UCI (coils/cm) & $0.268(0.13)$ & $0.101(0.04)$ & $0.254(0.06)$ & $0.549(0.14)$ & \\
\hline
\end{tabular}

$\mathrm{GDM}=$ gestational diabetes mellitus; NS = non-significant (by ANOVA/chi-squared test; normocoiled group as a reference); PIH = pregnancy-induced hypertension; SGA = small for gestation age; UCI = umbilical coiling index. Numerical values are expressed in mean (standard deviation); for categorical values frequency of occurrence is given.

For umbilical cord twist directions, we did not observe any direction to be an overtly predominant with $53 \%$ cords having twist in anticlockwise direction, while $47 \%$ having it in a clockwise manner. The two groups were 
found to have comparable demographic characteristic (Table 2).

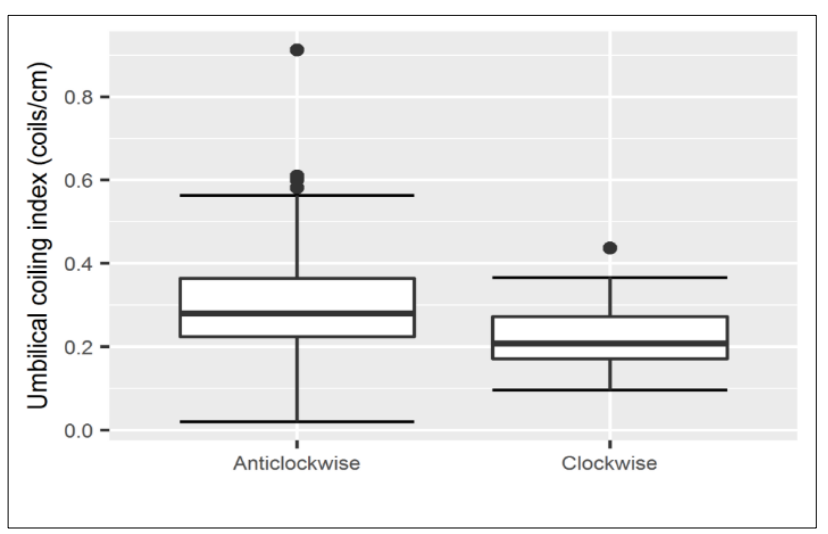

Figure 2: Distribution of umbilical coiling index (UCI) as per the umbilical cord twist directions.

However, we found a significant difference in UCI in these two groups with anticlockwise group having significantly high UCI; this difference was found to be statistically significant even after removing the outlier value $(\mathrm{UCI}=0.912)$. Figure 2 , shows a boxplot graph of UCI according to the twist directions.

\section{Association between pregnancy-related factors and UCI}

We studied the following risk factors, maternal age, GDM, PIH, oligohydramnios, SGA, and fetal gender for their association with abnormal cord coiling. Both maternal age (rho $=0.0094)$ and gestational age (rho $=$ 0.0144 ) were found to be very weakly correlated with UCI. We did not find any statistically significant association of these factors with hypocoiled or hypercoiled cord group with normocoiled cord group as a reference using multivariate logistic regression analysis (Table 3). As we had no case of GDM and oligohydramnios in hypocoiled cord group we could not define their relationship with hypocoiling.

Table 2: Demographic characteristics of the study population as per the cord twist directions.

\begin{tabular}{|llll|}
\hline Characteristic & Clockwise group $(n=47)$ & Anticlockwise group $(\mathrm{n}=53)$ & Statistical difference \\
\hline Maternal age (year) & $23.34(3.30)$ & $23.53(2.96)$ & NS \\
\hline Gestational age (week) & $38.52(1.13)$ & $38.17(1.27)$ & NS \\
\hline Birth weight $(\mathrm{g})$ & $2789.57(371.31)$ & $2735.44(429.22)$ & NS \\
\hline UCI (coils/cm) & $0.225(0.079)$ & $0.307(0.153)$ & Significant $(p=0.0015)$ \\
\hline
\end{tabular}

NS = non-significant (by Student's independent sample t-test); UCI = umbilical coiling index; Values are expressed in mean (standard deviation).

Table 3: Multivariate logistic regression analysis for association between pregnancy-related factors and the UCI categorizes.

\begin{tabular}{|lll|}
\hline Factor & Hypocoiled group (OR with 95\% CI) & Hypercoiled group (OR with 95\% CI) \\
\hline Maternal age (year) & $1.08(0.87-1.35)$ & $1.04(0.84-1.28)$ \\
\hline GDM (yes/no) & Undefined $^{\#}$ & $1.34(0.12-14.22)$ \\
\hline PIH (yes/no) & $0.86(0.09-8.11)$ & $0.74(0.07-6.93)$ \\
\hline Oligohydramnios (yes/no) & Undefined $^{\#}$ & $0.27(0.03-2.44)$ \\
\hline SGA (yes/no) & $5.05(0.96-26.34)$ & $3.37(0.28-39.77)$ \\
\hline Fetal gender (male/female) & $4.54(0.89-22.97)$ & $2.85(0.66-12.32)$ \\
\hline
\end{tabular}

$\mathrm{CI}=$ confidence interval; GDM = gestational diabetes mellitus; OR = odds ratio (with normocoiled group as a reference); PIH = pregnancy-induced hypertension; SGA = small for gestation age; \#No cases were present in this category.

Table 4: The UCI categories and the adverse perinatal outcomes.

\begin{tabular}{|lllllll|}
\hline Perinatal outcome & $\begin{array}{l}\text { Study } \\
\text { population }\end{array}$ & $\begin{array}{l}\text { Normocoiled } \\
\text { group }\end{array}$ & $\begin{array}{l}\text { Hypocoiled } \\
\text { group }\end{array}$ & $\begin{array}{l}\text { Statistical } \\
\text { difference }\end{array}$ & $\begin{array}{l}\text { Hypercoiled } \\
\text { group }\end{array}$ & $\begin{array}{l}\text { Statistical } \\
\text { difference }\end{array}$ \\
\hline Meconium staining & 11 & 9 & 1 & NS & 1 & NS \\
\hline $\begin{array}{l}\text { Non-reassuring FHR pattern on } \\
\text { CTG }\end{array}$ & 28 & 20 & 2 & NS & 6 & $\begin{array}{l}\text { Significant } \\
(\text { P }=0.0309)\end{array}$ \\
\hline Low Apgar $(<7)$ at 1 minute & 8 & 6 & 1 & NS & 1 & NS \\
\hline Low Apgar $(<7)$ at 5 minute & 0 & 0 & 0 & Undefined $^{\#}$ & 0 & Undefined $^{\#}$ \\
\hline LBW $(\leq 2500$ g) & 26 & 21 & 1 & NS & 4 & NS \\
\hline NICU admission & 31 & 26 & 3 & NS & 2 & NS \\
\hline
\end{tabular}

CTG = cardiotocography; FHR = fetal hear rate; LBW = low birth weight; NICU = neonatal intensive care unit; NS = nonsignificant (by Fisher's exact test with the normocoiled group as a reference); * Indicates statistically significant association; \#No cases were present in this category. 


\section{The UCI categories and adverse perinatal outcomes}

In regard to adverse perinatal outcomes, we examined six outcomes, meconium staining of the amniotic fluid, nonreassuring FHR, low Apgar score (one and five minutes), LBW newborns, and NICU admission. For the hypocoiled group, no statistically significant difference was observed in occurrence of adverse perinatal outcomes in comparison with the normocoiled group. However, for the hypercoiled group, a statistically significant high occurrence of non-reassuring FHR patterns on CTG was observed when compared to that of normocoiled group. All other outcomes were found to be statically non-significant (Table 4).

To quantify strength of association OR was computed for each perinatal outcome for hypocoiled and hypercoiled groups with normocoiled groups as a reference (Table 5). We could not compute OR for low Apgar at five minutes as our study population had no cases in this category. Based on ORs and their 95\% CI, we found that nonreassuring FHR patterns on CTG were significantly associated with the hypercoiled cord $(\mathrm{OR}=4.5 ; \mathrm{CI}=1.15$ - 17.58).

Table 5: Association between the UCI categories and adverse perinatal outcomes.

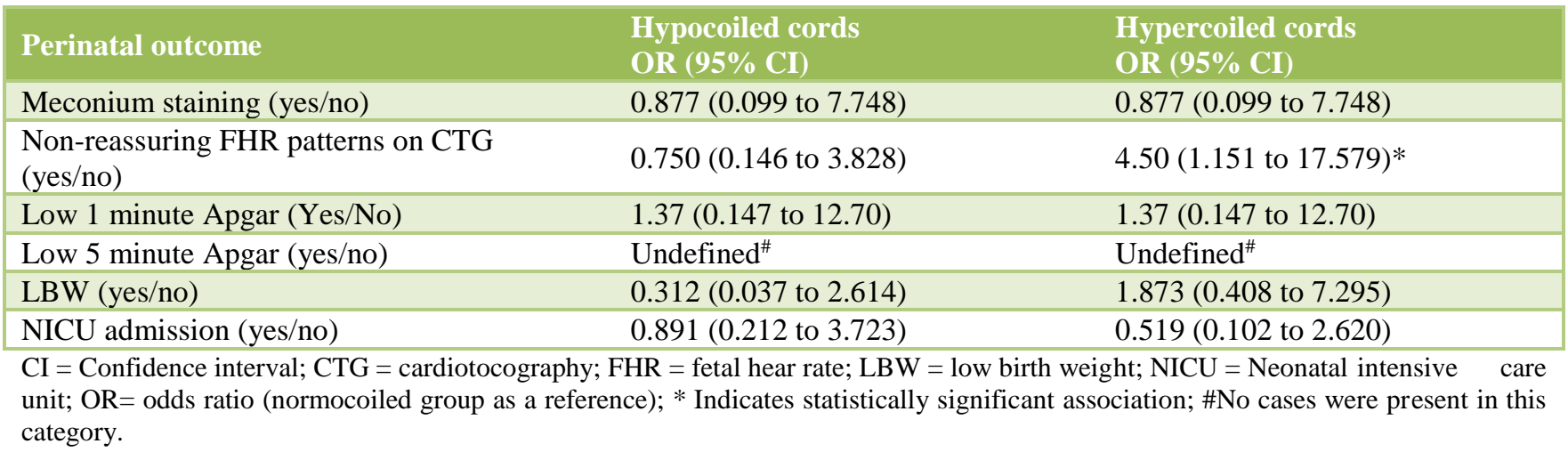

Table 6: Association between cord twist directions and the adverse perinatal outcomes.

\begin{tabular}{|llll|}
\hline Perinatal outcome & Clockwise group & Anticlockwise group & OR (95\% CI) \\
\hline Meconium staining (yes/no) & 7 & 4 & 2.14 (0.58 to 7.84) \\
\hline $\begin{array}{l}\text { Non-reassuring FHR patterns on CTG } \\
\text { (yes/no) }\end{array}$ & 12 & 16 & $0.79(0.32$ to 1.91$)$ \\
\hline Low 1 minute Apgar (yes/no) & 3 & 5 & $0.49(0.22$ to 1.09$)$ \\
\hline Low 5 minute Apgar (yes/no) & 0 & 0 & Undefined $^{\#}$ \\
\hline LBW (yes/no) & 12 & 14 & $0.95(0.39$ to 2.33) \\
\hline NICU admission (yes/no) & 15 & 16 & $1.08(0.46$ to 2.53) \\
\hline
\end{tabular}

$\mathrm{CI}=$ Confidence interval; $\mathrm{CTG}=$ cardiotocography; FHR $=$ fetal hear rate; $\mathrm{LBW}=$ low birth weight; NICU = Neonatal intensive care unit; OR = odds ratio; \#No cases were present in this category.

None of other outcomes was found to have any significant association with abnormal cord coiling.

\section{Umbilical cord twist direction and adverse perinatal outcomes}

In regard to umbilical cord twist direction we did not find any statistically significant difference between cord twist directions and the studied perinatal outcomes (Table 6). We could not compute OR for low Apgar at five minutes as our study population had no cases in this category.

\section{DISCUSSION}

This study is an attempt to study origin of umbilical cord characteristics and association of these characteristics with adverse perinatal outcomes in a systematic manner. This study had a few important findings in this regard: (1) out of all adverse perinatal outcomes, hypercoiling of cord was found to be significantly associated with nonreassuring or abnormal FHR patterns on CTG; (2) anticlockwise twisted cords had significantly high UCI compared to clockwise twisted cords; (3) no significant association was found between various pregnancy-related factors and origin of abnormal cord coiling.

In this study, a wide variation in UCI was observed; the mean UCI in this study was found to be higher compared to other studies, but within a limit of UCI reported from India. ${ }^{14}$ In general, we observed that studies on Indian population have reported higher UCI compared to the studies from the other countries, which have consistently reported 0.2 coils/cm as normal postnatal UCI. ${ }^{4,8,14}$ This 
could be due to already observed anthropometric difference between Indian and Western babies, and thus may call for thorough evaluation of umbilical cord characteristics from ethnic perspective as well. ${ }^{19}$ For a one particular case we observed 0.911 coils $/ \mathrm{cm}$ as an extreme value of UCI. This near-term case had history of severe PIH and antepartum haemorrhage. The woman had to be delivered by emergency caesarean section due non-reassuring FHR patterns on CTG. The neonate had low birth weight and low Apgar score at one minute and was admitted to the NICU. This particular case was marked with very short cord with length of $31 \mathrm{~cm}$. This point towards need of more research in this area to fully understand the pathophysiological significance of cord characteristics.

In regard to cord twist directions, a number of studies have reported predominance of anticlockwise direction. ${ }^{7,11,20}$ Although a number of theories from genetic patterns to forceful paddling with the right arm by right-handed fetus have been attributed to predominance of anticlockwise twist, none of these theories had been able to fully explain it yet. ${ }^{3,7}$ In our study, we observed only slight preponderance of anticlockwise cords over clockwise cords; which also highlights limitation of existing theories in fully explaining this phenomenon.

In this study, we examined association of pregnancyrelated factors with umbilical cord characteristics using multivariate logistic regression analysis. As a number of factors are shown to be associated with abnormal cord coiling, a multivariate logistic regression is recommended over bivariate analysis to study such association. ${ }^{1}$ However, no statistically significant association of these factors with hypocoiled or hypercoiled cord group was found in this study. In regard to six adverse perinatal outcomes studied, only the odds of occurring of nonreassuring or abnormal FHR patterns on CTG was found to be significantly higher in hypercoiled groups $(\mathrm{OR}=$ 4.5). This finding is consistent with a number of studies, which have found similar association..$^{15,21,22}$ We did not find any statistically significant difference between cord twist direction and the adverse perinatal outcomes.

Most of the existing studies have analyzed umbilical cord characteristic in isolation rather than studying their combined relationship with perinatal outcomes. In our study when relationship between UCI and cord direction was studied it was observed that anticlockwise twisted cords had significantly high UCI compared to clockwise twisted cords. Other studies have also reported similar findings; however, none of the authors have offered any explanation for this. ${ }^{7,11} \mathrm{We}$ further observed that out of 10 cases in hypercoiled cord group, nine had anticlockwise twisted cords and these cases were associated with majority of averse perinatal outcomes. Given this fact we recommend thorough investigation of association of combined umbilical cord characteristics with adverse perinatal outcomes.
The limitations of our study are that data was collected from a single center on a small number of samples; this makes it difficult to study association of all adverse outcomes and generalize these results. However, our study has shown results which are consistent with existing literature. The strength of our study lies in being the first study on Indian population where association between pregnancy-related factors and cord characteristics was systematically studied using multivariate regression analysis. Moreover, in this study we have also studied the relationship between UCI and cord twist direction, which is still an unexplored topic.

\section{CONCLUSION}

To conclude, very limited information is available from India on pathophysiological significance of umbilical cord anomalies and their implication in clinical practices; our study is an attempt to fill this gap in the existing literature. This study has also raised new questions regarding association between different umbilical cord characteristic put together with clinical outcomes and possible impact of anthropometric variations in different populations on cord characteristics. Considering these factor, we recommend further evaluation of these parameters in large multicenter studies.

\section{Funding: No funding sources}

Conflict of interest: None declared

Ethical approval: The study was approved by the Institutional Ethics Committee

\section{REFERENCES}

1. de Laat MWM, Franx A, van Alderen ED, Nikkels PGJ, Visser GHA. The umbilical coiling index, a review of the literature. J Matern-Fetal Neonatal Med Off J Eur Assoc Perinat Med Fed Asia Ocean Perinat Soc Int Soc Perinat Obstet. 2005;17(2):93-100.

2. Ernst LM, Minturn L, Huang MH, Curry E, Su EJ. Gross patterns of umbilical cord coiling: correlations with placental histology and stillbirth. Placenta. 2013;34(7):583-8.

3. Lacro RV, Jones KL, Benirschke K. The umbilical cord twist: origin, direction, and relevance. Am J Obstet Gynecol. 1987;157(4 Pt 1):833-8.

4. Sebire NJ. Pathophysiological significance of abnormal umbilical cord coiling index. Ultrasound Obstet Gynecol. 2007;30(6):804-6.

5. Kalish RB, Hunter T, Sharma G, Baergen RN. Clinical significance of the umbilical cord twist. Am J Obstet Gynecol. 2003;189(3):736-9.

6. Jessop FA, Lees CC, Pathak S, Hook CE, Sebire NJ. Umbilical cord coiling: clinical outcomes in an unselected population and systematic review. Virchows Arch Int J Pathol. 2014;464(1):105-12.

7. de Laat MWM, Franx A, Bots ML, Visser GHA, Nikkels PGJ. Umbilical coiling index in normal and complicated pregnancies. Obstet Gynecol. 2006;107(5):1049-55. 
8. Chitra T, Sushanth YS, Raghavan S. Umbilical coiling index as a marker of perinatal outcome: an analytical study. Obstet Gynecol Int. 2012;2012:e213689.

9. Ezimokhai M, Rizk DE, Thomas L. Maternal risk factors for abnormal vascular coiling of the umbilical cord. Am J Perinatol. 1999; 17(8):441-5.

10. Khong TY. Evidence-based pathology: umbilical cord coiling. Pathology (Phila). 2010;42(7):618-22.

11. Gupta S, MMA F. Umbilical coiling index. J Obstet Gynecol India. 2006;56(4):315-9.

12. Sharma B, Bhardwaj N, Gupta S, Gupta PK, Verma A, Malviya K. Association of umbilical coiling index by colour doppler ultrasonography at 18-22 weeks of gestation and perinatal outcome. J Obstet Gynecol India. 2012;62(6):650-4.

13. Patil NS, Kulkarni SR, Lohitashwa R. Umbilical cord coiling index and perinatal outcome. J Clin Diagn Res JCDR. 2013;7(8):1675-7.

14. Agarwal S, Purohit R, Jani G. Umbilical cord coiling index and perinatal outcome in normal and abnormal pregnancies. Sch J Appl Med Sci. 2014;447-50.

15. Strong TH, Jarles DL, Vega JS, Feldman DB. The umbilical coiling index. Am J Obstet Gynecol. 1994;170(1 Pt 1):29-32.

16. Rana J, Ebert GA, Kappy KA. Adverse perinatal outcome in patients with an abnormal umbilical coiling index. Obstet Gynecol. 1995;85(4):573-7.

17. Mikolajczyk RT, Zhang J, Betran AP, Souza JP, Mori R, Gülmezoglu AM, et al. A global reference for fetal-weight and birthweight percentiles. The Lancet. 2011;377(9780):1855-61.
18. Macones GA, Hankins GDV, Spong CY, Hauth J, Moore T. The 2008 National Institute of Child Health and Human Development workshop report on electronic fetal monitoring: update on definitions, interpretation, and research guidelines. J Obstet Gynecol Neonatal Nurs JOGNN. 2008;37(5):510-5.

19. Yajnik CS, Fall CHD, Coyaji KJ, Hirve SS, Rao S, Barker DJP, et al. Neonatal anthropometry: the thinfat Indian baby. The Pune Maternal Nutrition Study. Int J Obes Relat Metab Disord J Int Assoc Obes. 2003;27(2):173-80.

20. Pathak S, Hook E, Hackett G, Murdoch E, Sebire NJ, Jessop F, et al. Cord coiling, umbilical cord insertion and placental shape in an unselected cohort delivering at term: Relationship with common obstetric outcomes. Placenta. 2010;31(11):963-8.

21. Machin GA, Ackerman J, Gilbert-Barness E. Abnormal umbilical cord coiling is associated with adverse perinatal outcomes. Pediatr Dev Pathol. 2000;3(5):462-71.

22. Hasegawa J, Matsuoka R, Ichizuka K, Sekizawa A, Okai T. Ultrasound diagnosis and management of umbilical cord abnormalities. Taiwan J Obstet Gynecol. 2009;48(1):23-7.

Cite this article as: Sarkate PS, Hiwale S.

Relationship of umbilical coiling index and cord twist direction with adverse perinatal outcomes. Int $\mathbf{J}$ Reprod Contracept Obstet Gynecol 2019;8:3782-8. 